\title{
Tendencias temáticas en psicología publicadas por Psicogente en el período 2014-2017
}

\section{Subjects in psychology published by Psicogente between 2014 and 2017}
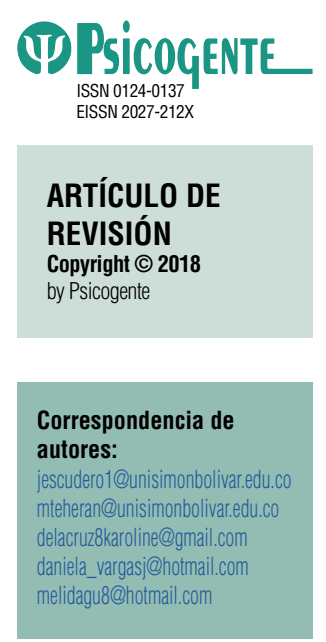

Recibido: $18-01-18$ Aceptado: $18-05-18$ Publicado: 01-07-18

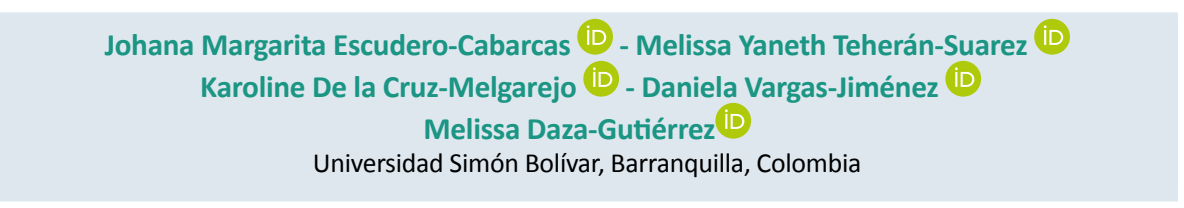

Resumen

Objetivo: Identificar las temáticas con mayor tendencia de publicaciones cientificas de la Revista Psicogente en los años 2014 a 2017 lo que permitirá a los investigadores y científicos tener una ruta orientativa de los campos psicológicos que se encuentra en mayor auge investigativo según el análisis bibliográfico de la revista.

Método: Artículo de revisión con diseño de tipo exploratorio de corte bibliográfico, se analizaron 111 artículos publicados por la Revista Psicogente con un sistema Open Access. Las variables analizadas fueron: temática de estudio, población y país de procedencia de cada uno de los artículos. A través de una matriz se agruparon las variables para identificar las tendencias con mayor frecuencia.

Resultados: Psicología social y clínica con un 38.7 \% y $19.4 \%$ respectivamente tienen mayor relevancia en publicación en la Revista Psicogente, los países de Colombia y México mostraron una mayor frecuencia en publicación con un $54.8 \%$ y $19.4 \%$ respectivamente y la población de estudiantes de secundarias con un $49.5 \%$ fue de mayor interés de los investigadores para sus publicaciones.

Conclusión: Las revisiones bibliográficas de las revistas científicas son una ruta orientativa para los investigadores y científicos que requieran un mapa claro de la investigación, en el caso de la Revista Psicogente, este artículo permite cumplir con el objetivo planteado, mostrando resultados claros y que permiten al lector científico una herramienta esencial a la hora de iniciar un proceso de investigación. Con la muestra y análisis de 111 artículos se evidencio que la psicología social en Colombia es una de las mayores tendencias en investigación científica.

\section{Palabras clave: Psicología, tendencias, investigación, revista científica, Psicogente.}

\section{Abstract}

Objective: To identify the topics with the highest tendency of scientific publications of the Psicogente in the years 2014 to 2017. This will allow researchers and scientists to have an indicative route of the psychological fields that is in the highest research boom according to the bibliographic analysis of journal.

Method: Review paper with bibliographic exploratory design. 111 articles published by the Psicogente with an Open Access system were analyzed. The variables analyzed were subject, population and country for each paper. The variables were organized on a matrix to identify the frequency of the trends.

Results: Social and clinical psychology with $38.7 \%$ and $19.4 \%$ respectively have greater relevance in publication in the psychogenic journal, the countries of Colombia and Mexico showed a greater frequency in publication with $54.8 \%$ and $19.4 \%$ respectively and the population of high school students with $49.5 \%$ was of greater interest to researchers for their publications.

Conclusion: Bibliographic reviews of scientific journals are an indicative route for researchers and scientists who require a clear map of the research, in the case of psychogenic journal, this article allows to meet the stated objective, showing clear results and allowing the scientific reader an essential tool when starting a research process. With the sample and analysis of 111 articles it was evidenced that social psychology in Colombia is one of the biggest trends in scientific research.

Key Words: Psychology, Trends, Research, journal, Psicogente.

Cómo citar este artículo (APA):

Escudero-Cabarcas, J. M., Teherán Suárez, M. Y., De la Cruz Melgarejo, K., Vargas-Jiménez, D. \& Daza-Gutiérrez, M2. (2018). Tendencias temáticas en psicología publicadas por Psicogente en el periodo 2014-2017. Psicogente 21(40), 560-585. https://doi.org/10.17081/psico.21.40.2705 


\section{INTRODUCCIÓN}

A medida que avanzan los años y se realizan diferentes estudios acerca de la Psicología, se hace cada vez más evidente el crecimiento que ésta ha tenido en materia de investigaciones. Dicho crecimiento se debe principalmente a la importancia que se le da a la divulgación de los hallazgos realizados a partir de las investigaciones científicas, lo que ha influido en la creación y consolidación de medios a través de los cuales se publican los artículos investigativos relacionados, en este caso, a la Psicología (López-López, Silva, García-Cepero, Aguilar-Bustamante \& Aguado-López, 2011).

Acorde a García, Guerrero, Vargas \& Moya-Anegón (2008), los países latinoamericanos en general realizan investigaciones en Psicología orientadas al área de las neurociencias, la psicología social y la psicología educativa. Según su estudio, Colombia explora temáticas relacionadas a la Neurología.

Especificando por área, en la investigación realizada por Rey-Anacona, Martínez-Gómez \& Guerrero-Rodríguez (2009), se encuentra que en los artículos científicos en psicología clínica de Iberoamérica publicados a través de los sistemas de información REDALYC y PSICODOC, hay una marcada tendencia a indagar temas relacionados a la psicopatología respecto a ansiedad, la psicoterapia cognitiva y conductual y la evaluación psicológica.

Posteriormente, Lilo \& Martini en el año 2013 indagan también acerca de las principales tendencias iberoamericanas en psicología clínica y encuentran que las investigaciones de esta área están encaminadas principalmente a la depresión y a la demencia-Alzheimer. Por otra parte, según su análisis, España produce $54 \%$ de la ciencia y lidera $44 \%$ de la producción más influyente citada mientras Latinoamérica produce $37 \%$ y lidera $13 \%$ (Brasil $10 \%$, Chile $1 \%$, Argentina $1 \%$, Colombia $1 \%$ ).

Respecto a la producción por países, Vera-Villarroel, López-López, Lillo \& Silva (2011) en su investigación sugieren que Brasil, México, Argentina, Chile y Colombia tienen los mejores indicadores en términos de producción e impacto, tanto en ciencia como en Psicología. Chile, Argentina y Brasil son los países más productivos por millón de habitantes.

En el año 2014, Reyes, Alarcón \& Bahamón realizaron una investigación acerca de las tendencias en líneas de investigación de doctorados en psicología de universidades iberoamericanas y sus resultados arrojaron que el área que 
más se explora es la Psicología Clínia y, además, el tema acerca del cual más se investiga es la cognición.

Aunque se encuentran análisis de tendencias respecto a la Psicología Clínica, no se obtuvo ningún resultado al indagar acerca de tendencias investigativas en otras áreas, lo cual influye en el objetivo de esta investigación, pues a través de ella se espera identificar y mostrar las principales tendencias en investigaciones científicas de Psicología publicadas en la Revista Psicogente de la Universidad Simón Bolívar durante el período 2014-2017, analizando las temáticas desarrolladas, el enfoque desde el cual se abordan, las poblaciones estudiadas y los países desde los que se realizan las investigaciones.

A través de esta revisión bibliográfica se pretende aportar un insumo en la contextualización y organización de tendencias de investigación en psicología con el fin de ofrecer un punto de referencia en el que encuentren ya identificadas las áreas y temáticas exploradas por otros, para así considerar por nuevos abordajes teóricos, replantear las hipótesis o suplir vacíos existentes en los artículos ya publicados.

\section{MÉTODO}

\subsection{Diseño}

Esta es una investigación de tipo exploratoria de corte bibliográfico, la cual se sustenta bajo Hernández, Fernández \& Baptista (2014), quienes plantean que este tipo de investigaciones sirven para examinar algún tema o problemática poco conocido en la actualidad, por lo que el corte exploratorio-bibliográfico aumenta el grado de conocimiento de dicho fenómeno con el propósito de ampliar el foco sobre ese campo y permita direccionar las prioridades para investigaciones posteriores. A su vez, la investigación cuenta con un diseño descriptivo que tiene como objetivo indagar modalidades en las variables en una población, a raíz de tal descripción se establecen diferentes hipótesis que son directivas en el proceso de investigar (Hernández, Fernández \& Baptista, 2014).

\subsection{Unidades de análisis e Instrumentos}

Se realiza una búsqueda de artículos en la OJS de la Revista Psicogente; como criterio de inclusión de tomaron todos aquellos artículos publicados en Psicogente entre los años 2014 y 2017. Se excluyeron editoriales, cartas 
a editor y reseñas. Cada uno de ellos fue direccionado según su tendencia a estudiar, diferenciándose por las múltiples áreas y temáticas que abordaron.

Las líneas temáticas encontradas fueron:

1. Psicología Social

2. Psicología Clínica

3. Neuropsicología

4. Psicología Educativa

5. Psicología Organizacional

6. Psicometría

7. Psicología Política

8. Psicología de Mercado

9. Ética

\subsection{Procedimiento}

Se inició con el proceso de revisión bibliográfica de los artículos de revisión y originales (con reportes de resultados), de la Revista Psicogente disponibles en el OJS desde el año 2014 hasta el 2017. Primero se definieron los años que se analizarían, seguido de la definición de variables que se tendrían en cuenta como datos descriptores: población, país de procedencia y el análisis principal, la temática de la psicologia en que se basó la investigación. Posterior a este paso se procedió a analizar por años las variables empleando estadística descriptiva.

\subsection{Análisis de resultados}

Posterior a la organización e identificación de las variables de análisis: temática del artículo, población y país de procedencia, se incorporó la información en el estadístico SPSS software que permite el análisis estadístico descriptivo como la tabulación y frecuencias de cruce, de las variables estudiadas, esto permitió obtener la información numérica y de base para interpretar los resultados de la investigación.

\subsection{Conflictos de interés}

Los autores declaran que en la realización del texto y recolección de la información no se presenta ningún conflicto de interés en el texto presentado para su publicación. 


\section{RESULTADOS}

Tabla 1.

Análisis estadístico de áreas de tendencia en el año 2014

\begin{tabular}{ccc}
\hline ÁREA EN TENDENCIA & $\begin{array}{c}\text { No. } \\
\text { ARTícUlos }\end{array}$ & PORCENTAJE \\
\hline TOTAL & 31 & \\
Psicología Social & 12 & 38.7 \\
Psicología Clínica & 6 & 19.4 \\
Psicometría & 4 & 12.9 \\
Psicología Educativa & 4 & 12.9 \\
Neuropsicología & 3 & 9.7 \\
Psicología Organizacional & 1 & 3.2 \\
Artículo Tendencia & 1 & 3.2 \\
\hline
\end{tabular}

Tabla 2.

Análisis estadísticos del número de artículos publicados por país en el año 2014

\begin{tabular}{ccc}
\hline PAís & No. ARTíCULOS & PORCENTAJE \\
\hline Colombia & 17 & $54.8 \%$ \\
México & 6 & $19.4 \%$ \\
Cuba & 3 & $9.7 \%$ \\
Argentina & 2 & $6.5 \%$ \\
El Salvador & 1 & $3.2 \%$ \\
España & 1 & $3.2 \%$ \\
Venezuela & 1 & $3.2 \%$ \\
\hline
\end{tabular}

Tabla 3.

Análisis de tipo de población y unidades de estudio utilizadas por los investigadores Psicogente año 2014

\begin{tabular}{ccc}
\hline $\begin{array}{c}\text { POBLACIÓN/UNIDADES DE } \\
\text { ESTUDIO }\end{array}$ & $\begin{array}{c}\text { TIPO DE } \\
\text { POBLACIÓN }\end{array}$ & PORCENTAJE \\
\hline Estudiantes Secundaria & 2666 & 49.5 \\
Docentes & 584 & 11 \\
Estudiantes Universitarios & 516 & 9.6 \\
Adultos & 501 & 9.3 \\
Vendedores & 389 & 7.2 \\
Niños & 345 & 6.4 \\
Amas de casa & 203 & 3.8 \\
Empleados & 76 & 1.4 \\
Pacientes & 71 & 1.3 \\
Líderes & 36 & 0.7 \\
\hline
\end{tabular}

En las tablas 1, 2 y 3 podemos encontrar los datos correspondientes al análisis realizado en el año 2014 de las variables: país, temática tendencia y población de estudio; se publicaron un total de 31 artículos, siendo psicología social la mayor área estudiada; el país donde más investigaciones se hicieron 
fue Colombia con un 54.8 \%. La población más estudiada fueron estudiantes de secundaria

En el año 2014 la Revista Psicogente publicó 31 artículos desde diferentes áreas de la psicología; se trataron temas enfocados en psicometría, psicología social, psicología educativa, psicología clínica, neuropsicología y psicología organizacional.

Dentro de los campos que más relevancia se otorgó en la Revista Psicogente durante este año fue la psicología social, ya que fueron publicados $38.7 \%$ de artículos; en este enfoque, su tendencia fue de estudios dirigidos a la relación entre los individuos y su grupo o entorno y a su vez se le dio relevancia a los factores psicosociales que influyen en dicha interacción (Marín, 2014)(Morales, 2014)(Rodríguez, Roque \& Rodríguez, 2014)(Campo, 2014)(Orozco, 2014) (Diazgranados \& Sánchez, 2014)(Muñoz, 2014)(Pérez, Vianchá, Martínez \& Salas, 2014) (Valdez \& Iranzo, 2014) (Cervantes, Acosta, Balaguera, Valenzuela \& Torres, 2014)(Rodríguez, Trillos \& Baute, 2014) (Juárez \& Landero, 2014). Haciendo una revisión, se encuentra que por estar relacionado a ámbitos sociales la población que se estudió fue muy variada, desde familias, amas de casa, vendedores informales, jóvenes universitarios y hasta docentes; sin embargo, se resalta que los adolescentes escolarizados fue la muestra más representativa dentro de los artículos desde este enfoque de la psicología. A su vez, se destaca que el lugar con mayores estudios fue la región Caribe, encabezada por la costa Atlántica y el Magdalena; sin embargo se encuentran estudios en el oriente del país, con investigaciones sociales en Tunja, Duitama y Sogamoso.

La segunda área con mayor número de publicaciones fue psicología clínica; contó con un $19.3 \%$ de artículos. Se investigaron temas desde el psicoanálisis y la estética corporal (Mass, 2014), las creencias irracionales (Álvarez, 2014), la calidad de vida relacionada con la ansiedad, la depresión y el optimismo (Pérez \& Otero, 2014), los efectos de las experiencias anómalas (Parra \& Corbetta, 2014), la personalidad como incidencia en la elección de una línea teórica (Cariaga \& Casari, 2014) y la aplicabilidad de los sistemas autorreferenciales que fueron configurando un fundamento ontológico y epistemológico de configuraciones (Salcedo \& Ortiz, 2014). Para el estudio de esta área se contó con estudiantes de psicología, adolescentes y pacientes histriónicos y con lupus. Por último se menciona que los países estudiados fueron Colombia, Argentina y Cuba. 
Por otro lado, el área de psicometría obtuvo el 12.9 \% de publicaciones, contando con revisiones en la escala de satisfacción de vida (Luna \& Laca, 2014); se estudiaron las relaciones de poder en el aula (Austria \& Castañeda, 2014); así mismo se hicieron estudios sobre la validez de la escala de sociotropía y autonomía (SAS) y cómo esta se adapta a los estándares colombianos (Toro \& Vargas, 2014). Por último, a través de un estudio instrumental se logró evaluar la confiabilidad del Inventario para la Medición del Engagement en el Trabajo (IMET) (Cárdenas \& Jaik, 2014). Los partícipes de gran parte de los estudios estaban relacionados con ámbitos académicos, siendo los estudiantes los más investigados; se agrega que dichas investigaciones fueron escritas desde los países de Colombia y México.

Al igual que el área anterior, la psicología educativa contó con un $12.9 \%$ de publicaciones dentro de la revista. Se realizaron estudios encaminados en el abordaje de referente teórico-metodológico para garantizar la efectividad de las acciones preventivas hacia la drogadicción y el tabaquismo (García, Molerio \& Pedraza, 2014); para este investigación se contó con la participación de jóvenes estudiantes desde el país de Cuba. En otra publicación se midieron los intereses vocacionales a partir de la prueba Kunder (Arévalo \& Cano, 2014) para este, la población fue de 88 estudiantes de psicología en El Salvador. Además, se investigó la incidencia de la educación inicial en la construcción de la subjetividad, siendo los partícipes niños y niñas de tres a cinco años en la ciudad de Bogotá, Colombia (Guzmán \& Vargas, 2014). Por último se realiza una verificación de cómo la metodología eLearning puede ayudar en la formación del aprendizaje a personas con trastorno del espectro autista. Este estudió fue escrito desde España (Fernández \& Miralles, 2014).

Se destaca que desde el enfoque de la neuropsicología se obtuvieron investigaciones que ocupan un $9.6 \%$, los temas tratados fueron las alteraciones neuropsicológicas en adolescentes infractores en la ciudad de Sincelejo, Sucre, Colombia (Ramírez \& Arroyo, 2014), y otras direccionadas a los procesos cognitivos implicados en la comprensión lectora: uno trataba sobre la precisión y velocidad (Torres \& Granados, 2014) y el otro sobre las estrategias metacognitivas y la motivación que influían en el proceso (Guerra, Guevara \& Robles, 2014). Estas últimas fueron realizadas desde México.

Desde el área de la psicología organizacional se abordaron los temas de motivación y satisfacción laboral como recurso para el cambio organizacional (García \& Forero, 2014), esto equivale un 3.2 \%, la población estudiantil fue de 76 empleados de diferentes empresas en la ciudad de Bogotá, Colombia. 
Para hacer un cierre, se menciona que la Revista Psicogente publicó un $3.2 \%$ dirigido a un artículo en tendencia, en el cual se analizaron las línea de investigación de 32 universidades de Iberoamérica que cuentan con programa de doctorado en Psicología.

Tabla 4.

Análisis de temáticas tendencias de los artículos del año 2015

\begin{tabular}{ccc}
\hline ÁREA EN TENDENCIA & No. ARTícULOS & PORCENTAJE \\
\hline Psicología Social & 7 & 23 \\
Psicología Clínica & 6 & 20 \\
Psicología Educativa & 4 & 13 \\
Psicología Organizacional & 4 & 13 \\
Psicología Cognitiva & 3 & 10 \\
Psicometría & 2 & 7 \\
Análisis de Política & 1 & 3 \\
Ética & 1 & 3 \\
Neuropsicología & 1 & 3 \\
Psicología Política & 1 & 3 \\
\hline
\end{tabular}

Tabla 5.

Análisis estadísticos del número de artículos publicados por país en el año 2015

\begin{tabular}{ccc}
\hline PAís & No. ARTíCULOS & PORCENTAJE \\
\hline Colombia & 15 & $50 \%$ \\
Argentina & 5 & $17 \%$ \\
México & 5 & $17 \%$ \\
Venezuela & 2 & $7 \%$ \\
Brasil & 1 & $3 \%$ \\
Chile & 1 & $3 \%$ \\
Cuba & 1 & $3 \%$ \\
\hline
\end{tabular}

Tabla 6.

Análisis de tipo de población y unidades de estudio utilizadas por los investigadores Psicogente año 2015

\begin{tabular}{ccc}
\hline POBLACIÓN/UNIDADES DE ESTUDIO & TIPO DE POBLACIÓN & PORCENTAJE \\
\hline Estudiantes Secundaria & 2995 & $71.8 \%$ \\
Pacientes & 552 & $13.2 \%$ \\
Adultos & 368 & $8.8 \%$ \\
Estudiante Universitario & 51 & $1.2 \%$ \\
Profesores - Directivos & 50 & $1.2 \%$ \\
Familias & 50 & $1.2 \%$ \\
Estudiantes Egresados & 41 & $1.0 \%$ \\
Ancianos & 30 & $0.7 \%$ \\
Psicólogos & 29 & $0.7 \%$ \\
Revisión bibliográfica & 4 & $0.1 \%$ \\
Disputas & 2 & $0.0 \%$ \\
DSM & 1 & $0.0 \%$ \\
\hline
\end{tabular}


En las tablas 3, 4 y 5 podemos observar que los artículos publicados fueron 30, siendo la Psicología Social la mayor área de tendencia; fueron escritos en su mayoría en Colombia; su población con más estudio fue estudiantes de secundaria.

En el año 2015, desde el campo de la Psicología Social se desarrolló la mayor parte de las publicaciones. Respecto a las tendencias de investigación, se encuentra que los autores indagaron acerca de temas como violencia psicosocial y sus repercusiones en la salud mental en habitantes de las ciudades con mayor reporte de hechos violentos en el estado de Coahuila, México (Chávez, Petrzelová, Zapata, Hernández \& Rodríguez, 2015), conflicto vecinal entre un hombre y una mujer y sus patrones de interacción relacionados al género en un estudio de caso (Angeli, 2015), migración y adaptación cultural en 14 estudiantes universitarios migrantes (Sosa \& Zubieta, 2015), subjetividades y territorialidades desde la cartografía social en el contexto de documentos colombianos (Moreno, Rojas, Fagua, Sanabria, Sanabria, \& Rey, 2015), comportamientos prosociales en adolescentes colombianos (Redondo, Rangel \& Luzardo, 2015), autogestión en agentes comunitarios de Nariño en Colombia (Zambrano, Aroca \& Rosero, 2015) y factores psicosociales presentes en desnutrición crónica en niñas y niños escolarizados (Paredes, 2015). Las investigaciones se realizaron principalmente desde Colombia, seguido por Argentina y México.

En el campo de la Psicología Clínica, los investigadores realizaron estudios respecto a discriminación en la familia a mujeres diagnosticadas con VIH (Moral \& Segovia, 2015), caracterización del autoconcepto en estudiantes universitarias (Pinilla, Montoya \& Dussán, 2015), aprecio a la belleza como fortaleza del carácter en adultos venezolanos (Garassini \& Valle, 2015), autoestima, autonomía funcional y apoyo social en adultos mayores institucionalizados (Guerrero-Martelo, Galván, Vásquez, Lázaro \& Morales, 2015) y fundamentos teóricos, conceptuales y científicos del DSM (Barreira, 2015). La mayoría de las publicaciones corresponden a Colombia, seguido por Argentina, México y Venezuela.

En lo correspondiente a la Psicología Organizacional, se divulgaron las exploraciones desarrolladas en referencia a la evaluación de la calidad de vida laboral relacionadas al género en profesionales de la salud (González, y otros, 2015), estrés laboral y salud ocupacional en psicólogos clínicos (Spiendler, Carlotto, Ogliari \& Giordani, 2015), relación entre socialización organizacional y compromiso organizacional en trabajadores (Calderón, Laca, Pando 
\& Pedroza, 2015) e inclusión laboral e imaginarios sociales en la población sorda (Morales, 2015). Las investigaciones de este enfoque fueron desarrolladas desde México, continuando Brasil y Chile.

Desde la Psicología Educativa, fueron publicados artículos referentes a creencias epistemológicas sobre las matemáticas y su relación con los métodos de enseñanza en profesores de secundaria básica (Vizcaíno \& Manzano, 2015), hábitos y técnicas de estudio en el ámbito universitario (Enríquez, Fajardo \& Garzón, 2015), factores de riesgo y protectores del bullying escolar en estudiantes con bajo rendimiento (Ferrel, Cuan, Londoño \& Ferrel, 2015) y competencias académicas en el proceso educativo y formativo en psicología en estudiantes y egresados (Yáñez \& Ortíz, 2015). Dichas publicaciones fueron desarrolladas en Colombia, seguidas por Cuba.

Respecto al área de la Psicología Cognitiva, las investigaciones publicadas se centraban en temas como el rendimiento cognitivo en adolescentes con VIH (Pino, 2015), nivel educativo en madres y su relación con el rendimiento cognitivo infanto-juvenil (Labín, Taborda \& Brenlla, 2015) y aprendizaje situado, sus escenarios y sus contenidos en estudiantes de secundaria (Páramo, Hederich, López, Sanabria \& Camargo, 2015). Las investigaciones fueron realizadas principalmente en Colombia, seguidas de Argentina.

En el campo de la Psicometría, fueron divulgados artículos en relación a la validez y confiabilidad de la escala de salud mental positiva aplicada a trabajadores de áreas del sector salud (Martínez, Pando, León, González, Aldrete \& López, 2015); consideraciones y reflexiones acerca de la versión colombiana de la escala Barratt de impulsividad para niños (Chahín-Pinzón, 2015) y validación de la escala de aprendizaje regulado en adolescentes (ElviraValdés \& Pujol, 2015). Dichas investigaciones fueron desarrolladas en México, Colombia y Venezuela, respectivamente.

Por último, también fueron publicadas investigaciones de Neuropsicología, como la de Abrahan \& Justel (2015), acerca de la músicoterapia y la neurorrehabilitación; de Psicología Política, referente a la relación entre la política y el psicoanálisis (Salcedo, 2015); de Ética, respecto al libro colectivo Experticia, sabiduría y desarrollo moral (Trujillo, 2015) y de Análisis de políticas, en un estudio acerca de sus alcances en materia de ciencia, tecnología e innovación en Atlántico, Colombia (Pérez, 2015). Las anteriores publicaciones fueron realizadas desde Argentina y Colombia, respectivamente. 
Tabla 7.

Análisis de temáticas tendencias de los artículos del año 2016

\begin{tabular}{ccc}
\hline ÁREA EN TENDENCIA & No. ARTíCULOS & PORCENTAJE \\
\hline Psicología Clínica & 6 & 25.0 \\
Psicología Educativa & 5 & 20.8 \\
Psicología Social & 4 & 16.7 \\
Psicología Organizacional & 3 & 12.5 \\
Psicometría & 3 & 12.5 \\
Artículo en Tendencia & 1 & 4.2 \\
Neuropsicología & 1 & 4.2 \\
Psicología del Mercado & 1 & 4.2 \\
\hline
\end{tabular}

Tabla 8.

Análisis estadísticos del número de artículos publicados por país en el año 2016

\begin{tabular}{ccc}
\hline PAís & No. ARTíCULOS & PORCENTAJE \\
\hline Colombia & 16 & 66.7 \\
México & 4 & 16.7 \\
Chile & 2 & 8.3 \\
Cuba & 1 & 4.2 \\
Brasil & 1 & 4.2 \\
\hline
\end{tabular}

\section{Tabla 9.}

Análisis de tipo de población y unidades de estudio utilizadas por los investigadores Psicogente año 2016

\begin{tabular}{ccc}
\hline POBLACIÓN/UNIDADES DE ESTUDIO & TIPO DE POBLACIÓN & PORCENTAJE \\
\hline Niños & 372 & 25.0 \\
Estudiantes Secundaria & 325 & 21.9 \\
Adultos & 284 & 19.1 \\
Estudiantes Universitarios & 266 & 17.9 \\
Deportistas & 205 & 13.8 \\
Docentes-Directivos & 20 & 1.3 \\
Estudiantes egresados & 8 & 0.5 \\
Revisión bibliográfica & 6 & 0.4 \\
\hline
\end{tabular}

En las tablas 7, 8 y 9 podemos observar que la Psicología Clínica cuenta con un $25 \%$ de publicaciones, siendo esta la de mayor abordaje; Colombia fue el país con mayores investigaciones y los niños son la población escogida para adentrarse a las temáticas.

Siguiendo con la exposición de los resultados, se encuentra que en el año 2016 se realizaron 24 publicaciones, existiendo artículos desde el área de la Psicología Organizacional, Psicología Social, Psicología Educativa, Psicología Clínica, Psicología del Mercado, Psicometría, artículos en tendencias y el área de Neurociencia. 
El área con mayor número de publicaciones fue Psicología Clínica, contando con un $25 \%$ de artículos en el año 2016. En este enfoque, se estudiaron las variables psicológicas de las personas con el virus del chikungunya (Romero, Rojas, Abad \& Gómez, 2016), para ello se escogió una muestra de 41 mujeres y 22 hombres que estuviesen afectados por el virus en la ciudad de Barranquilla, Colombia, a su vez se identificaron las características psicológicas relacionadas con el rendimiento en deportes conjuntos y su relación con el género, se estudiaron 205 deportistas entre hombres y mujeres. El lugar donde se realizó fue en Quindío, Colombia (Arias, Cardoso, Aguirre \& Arenas, 2016). Por otra parte, se reconoció el nivel de resiliencia entre estudiantes universitarios y se realizó una comparación en relación con sus carreras profesionales, para ello se contó con la participación de 141 estudiantes universitarios en la ciudad de Jalisco, México (Caldera, Aceves \& Reynoso, 2016). En este año se encuentra una lectura relacionar que focaliza el sistema de identidad personal como utilidad en la ampliación del self dialógico, investigación estructurada desde Chile (Andreucci \& Tartakowsky, 2016), a su vez, se propuso la intervención psicoeducativa como terapia efectiva para el cáncer de mama realizado desde Cuba (Montiel \& Guerra, 2016). Por último, se realizó una identificación literaria de los principales riesgos suicidas en estudiantes universitarios con el objetivo de aportar técnicas preventivas; fue realizado desde Colombia (Castañeda, 2016).

Le sigue la Psicología Educativa, con un 20.8 \% de publicaciones; se plantea un artículo de reflexión sobre resignificación del proceso de aprendizaje para el programa de enfermería en Santiago de Chile (Rivera, 2016); se postula la hipótesis como herramienta para el pensamiento científico; se estudiaron 44 niños y niñas entre cuatro y ocho años de edad en la ciudad de Bogotá, Colombia (Collantes \& Escobar, 2016). Se realiza un aprendizaje por parte de los directivos para la gestión y el mejoramiento de su función, en la cual participan 16 directivos, 10 hombres y 6 mujeres en el estado de Durango, México (Ortega, 2016). A su vez se indaga la incidencia de los factores asociados al desarrollo de la comprensión lectora; se cuenta con 30 estudiantes entre 10 y 18 años, 25 padres de familia y 10 docentes (Franco, Cárdenas \& Santrich, 2016), y por último, se estudia el estado cognitivo para el aprendizaje de una población de 292 niños y niñas en Cauca, Colombia (Serrano \& Angarita, 2016).

El área de Psicología Social cuenta con un $16.6 \%$ de artículos publicados; se establece una concepción de resistencia por parte de las escuelas a la reflexión sobre la composición de familias homomaternal (Arrial \& Neves, 
2016); se plantearon procesos de integración social a partir de redes de apoyo, formación académica y trabajo (Sanz, Moreno \& Pérez, 2016), sus partícipes fueron ocho jóvenes que habían estado privados de su libertad en la ciudad de Cali, Colombia. Se indaga sobre el apoyo social y su conexión con la comunicación cara a cara y la comunicación mediada por la tecnología; se evaluaron a 125 estudiantes entre los 17 y 25 años, en la ciudad de Barranquilla, Colombia (Madariaga \& Lozano, 2016). Se realiza una revisión documental en torno al tema de observatorio y observatorio social (Moreno \& Mantilla, 2016).

La Psicología Organizacional cuenta con el $12.5 \%$ de publicaciones; fue realizada una investigación bibliográfica sobre la relación existente entre las creencias irracionales, la adicción al trabajo y el síndrome de Burnout en el contexto de las organizaciones, en la ciudad de Barranquilla, Colombia (Polo, Santiago, Navarro \& Alí, 2016). Siguiendo esta línea organizativa se planteó la cultura sobre seguridad y salud ocupacional (Aurioles \& Torres, 2016) en la que participaron 20 asociados y 20 trabajadores en México y como cierre se cuenta con un estudio sobre la sobrecarga laboral y su relación con el ingreso salarial; para ello se cuenta con la participación de 100 cuidadores informales de la ciudad de Bucaramanga, Colombia (Cerquera, Pabón, Granados \& Galvis, 2016).

Con respecto a la Validación Psicométrica, se cuenta con un $12.5 \%$ de publicaciones. Dentro de esta área se evalúa la atención y los procesos metacognitivos a partir de la escala Observación de Estrategias Cognitivas y Metacognitivas; la muestra fue de 36 niños de cuatro a cinco años en el departamento de Magdalena, Colombia (Iguarán, Anaya, Paba \& Obispo, 2016). A su vez se hizo la validación y confiabilidad del instrumento de vínculo parental, con el objetivo de medir la percepción, la actitud y la conducta de los padres hacia sus hijos, con 125 participantes varones en el país de México (Robles, Oudhof \& Mercado, 2016). Por último, se comprueban las propiedades psicométricas del Cuestionario de Motivos del Consumo de Alcohol (CMC), en la que participaron 206 estudiantes de sexto a noveno grado de un colegio público en la ciudad de Bogotá, Colombia (Pardo, Bernal, Ávila \& Muñoz, 2016).

El área de Neurociencia contó con un $4.1 \%$ de publicaciones; desde este enfoque se cuenta con un artículo de revisión donde se trabajaron las estrategias y didácticas aplicadas a la enseñanza de las finanzas. Este fue realizado desde Barranquilla, Colombia (Rico \& Puentes, 2016). 
Se cuenta con un $4.1 \%$ de publicación para la psicología de mercado, donde se evalúa el comportamiento del consumidor y percepción del servicio, participaron 90 estudiantes de administración de empresa en la ciudad de Barranquilla - Colombia (Henríquez, Rada \& Jassir, 2016)

Este año fue publicado un artículo en tendencia (4.1\%) que expone un análisis bibliométrico de los trabajos de investigación de pregrado y posgrado que sobre los problemas de comportamiento externalizado en niños y adolescentes en Bogotá, Colombia (Moreno, Hernández \& Suárez, 2016).

Tabla 10

Análisis de temáticas tendencias de los artículos del año 2017

\begin{tabular}{ccc}
\hline ÁREA EN TENDENCIA & No. ARTíCULOS & PORCENTAJE \\
\hline Psicología Social & 10 & 38.5 \\
Psicología Clínica & 7 & 26.9 \\
Psicología Educativa & 4 & 15.4 \\
Psicología Forense & 2 & 7.7 \\
Neuropsicología & 1 & 3.8 \\
Psicología Cognitiva & 1 & 3.8 \\
Psicología Organizacional & 1 & 3.8 \\
\hline
\end{tabular}

Tabla 11.

Análisis estadísticos del número de artículos publicados por país en el año 2016

\begin{tabular}{ccc}
\hline PAís & No. ARTíCULOS & PORCENTAJE \\
\hline Colombia & 22 & 85 \\
Argentina & 2 & 8 \\
México & 1 & 4 \\
Brasil & 1 & 4 \\
\hline
\end{tabular}

Tabla 12.

Análisis de tipo de población y unidades de estudio utilizadas por los investigadores de Psicogente año 2016

\begin{tabular}{ccc}
\hline POBLACIÓN/UNIDADES DE ESTUDIO & TIPO DE POBLACIÓN & PORCENTAJE \\
\hline Estudiantes Universitarios & 1937 & 30.8 \\
Padres de Familia & 1600 & 25.44 \\
Estudiantes Primaria y Secundaria & 1586 & 25.2 \\
Víctimas de Homicidio & 310 & 4.9 \\
Niños & 294 & 4.7 \\
Adultos & 238 & 3.8 \\
Guardas de Seguridad & 113 & 1.8 \\
Entrevistas Policiales & 81 & 1.3 \\
Jóvenes & 65 & 1.0 \\
Instituciones Educativas & 47 & 0.7 \\
Profesores & 12 & 0.2 \\
Revisión Bibliográfica & 4 & 0.1 \\
\hline
\end{tabular}


En las tablas 10, 11 y 12 el área de tendencia con mayor número de publicaciones fue Psicología Social con un 38.5\%, Colombia fue el país con más número de investigaciones y la población más abordada fueron estudiantes universitarios.

En el año 2017 la Revista Psicogente de la Universidad Simón Bolívar publicó 26 artículos de diferentes enfoques tales como el área de la Psicología Clínica, Social, Organizacional, Educativa y Neuropsicología.

Teniendo en cuenta las tendencias dentro de los campos estudiados con mayor profundidad en los artículos se encuentra que el $38.4 \%$ de las investigaciones fueron realizadas desde el enfoque de la Psicología Social, abordando temas tales como los roles dentro de la familia, la autopercepción de la eficacia de los condones teniendo en cuenta el sexo (hombre-mujer), violencia de género hacia docentes, la resiliencia en personas divorciadas según su nivel educativo y económico, la correlación de actitud hacia la homosexualidad femenina y masculina entre estudiantes de medicina, la relación entre cohesión familiar y sintomatología depresiva, y por último la atención psicosocial a las personas víctimas de conflicto armado (FariasCarracedo \& Marlon, 2017) (Uribe, Riaño, Bonilla, Carrillo, Hernández \& Bahamón, 2017) (Gamboa, Ortiz \& Muñoz, 2017) (Campo-Arias, Herazo \& Oviedo, 2017) (Zambrano, Perugache, \& Figueroa, 2017) (Buitrago, Pulido \& Güicha-Duitama, 2017) (Cerquera, Peña, Garcia, Orejuela \& García, 2017). Teniendo en cuenta que la población de estas investigaciones se estudian desde el ámbito social son muy variadas desde adolescentes, jóvenes universitarios, docentes, personas víctimas de secuestro; se denota que la población más estudiada entre estas fue 308 estudiantes jóvenes universitarios con edades que oscilan entre los 19 a 26 años de edad. A su vez los lugares de más estudio fue encabezada por la región Andina; sin embargo también hubo otros lugares internacionales de estudio tales como Argentina.

La segunda área con mayor número de publicaciones fue la Psicología Clínica, contó con un 34,6 \% investigando temas como aspectos epidemiológicos relacionados con los homicidios, relación entre riesgo suicida, autoestima, desesperanza, estilos de socialización parental, análisis de denuncia de delitos sexuales, cirugía estética en mujeres y su relación con los esquemas desadaptativos tempranos, estrategias de afrontamiento en situaciones de 
miedo, consumo de sustancias psicoactivas, automutilación, y dependencia funcional y crónica en el adulto mayor (Mebarak y otros, 2017) (Andrade \& Gonzáles, 2017) (Gutiérrez de Piñeres, 2017) (Moretti \& Casari, 2017) (Reyes, Reséndiz, Alcázar \& Reidl, 2017) (Córdoba-Paz \& Betancourth-Zambrano, 2017) (Cerquera, Uribe, Matajira \& Correa, 2017) (Fortes \& Kother, 2017). El mayor número de población fue de 821 estudiantes de bachillerato de $9^{\circ}, 10^{\circ}$ y $11^{\circ}$; pero también hicieron parte niños, niñas, adolescentes, jóvenes universitarios, funcionarios públicos, y personas informales. A su vez, los lugares de más estudio fue encabezada nacionalmente por Colombia entre Bogotá, Medellín, Cúcuta, Atlántico y Magdalena, e internacionalmente países como Brasil, México y Argentina.

La tercera área con mayor número de publicaciones fue la Psicología Educativa con un total de 19.2 \% tratando temas tales como Relación entre los docentes-estudiantes de preescolar, violencia en contextos escolares, desempeño lector en niños con TDAH, influencia en movimientos sádicos en el rendimiento académico de los estudiantes, síndrome de Asperger y síndrome de Burnout (Betancourth, Burbano \& Venet, 2017) (Gamboa, Ortiz \& Muñoz, 2017) (Cardona \& Varela, 2017) (Rincón, Hernández, \& Prada, 2017) (Beltrán, Díaz \& Zapata, 2017) (Ferrel, Ferrel, Cantillo, Jaramillo \& Jiménez, 2017). La población estudiada va desde niños, adolescentes, jóvenes, docentes y padres de familia, siendo la mayor población entrevistada 1.028 estudiantes de la Universidad Estatal de Colombia. A su vez el lugar donde fueron abordadas y realizadas dichas investigaciones fue Colombia.

Por último las aéreas con menor número de publicaciones fueron desde el enfoque Neuropsicológico con un $3.8 \%$ con un solo tema investigativo titulado Filogenia y desarrollo de las funciones ejecutivas (Calle, 2017) con una población de 37 menores con edades que oscilan entre los 4 y 5 años de edad, desarrollado en Colombia.

Desde el enfoque de la Psicología Organizacional obtuvo, al igual que el área anterior, un 3.8 \% con un único tema, Relación entre las diferentes dimensiones del Síndrome de Burnout y las estrategias de afrontamiento empleadas por los guardias de seguridad de una empresa privada de la ciudad de Tunja (Cruz \& Puentes, 2017) siendo la población estudiada y el lugar de abordaje Colombia. 
Tabla 13.

Análisis de tendencias de la Revista Psicogente 2014-2017

\begin{tabular}{ccc}
\hline ÁREA EN TENDENCIA & No. ARTícULOS & PORCENTAJE \\
\hline Psicología Social & 33 & 29.7 \\
Psicología Clínica & 25 & 22.5 \\
Psicología Educativa & 17 & 15.3 \\
Psicometría & 9 & 8.1 \\
Psicología Organizacional & 9 & 8.1 \\
Neuropsicología & 6 & 5.4 \\
Psicología Cognitiva & 4 & 3.6 \\
Psicología Forense & 2 & 1.8 \\
Artículo en Tendencia & 2 & 1.8 \\
Psicología del Mercado & 1 & 0.9 \\
Ética & 1 & 0.9 \\
Análisis de Política & 1 & 0.9 \\
Psicología Política & 1 & 0,9 \\
\hline
\end{tabular}

En la Tabla 13 podemos observar que el área en tendencia más estudiada es Psicología Social con un total de 29.7 \% de publicaciones, el país donde fueron realizados es Colombia con un porcentaje de $63 \%$.

Se concluye que en los períodos correspondientes al 2014, 2015, 2016 y 2017 fueron publicados 111 artículos, donde al integrar los temas objeto de abordaje se manifiesta el área de Psicología Social como tendencia mayor-

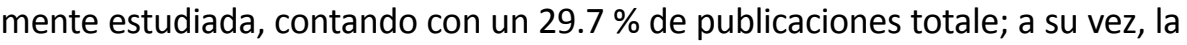
psicología clínica abarca un amplio número de investigaciones con un $22.5 \%$.

\section{DISCUSIÓN}

Llevar a cabo un análisis respecto a los artículos publicados en la Revista Psicogente de la Universidad Simón Bolívar durante el período 2014-2017 implica un ejercicio analítico mediante el cual se identifican las tendencias e intereses investigativos de los autores desde las diferentes áreas de la Psicología, las poblaciones estudiadas y los países que cuentan con mayor representación investigativa.

Respecto a la distribución de publicaciones por países, los resultados del presente estudio no se encuentran en coherencia con los hallazgos de la investigación realizada por Vera-Villarroel, López-López, Lillo, \& Silva (2011), pues mientras que dicho estudio encontró a Chile, Argentina y Brasil como los países más productivos, la presente investigación identificó como tal a 
Colombia, México y Argentina. Los resultados de la investigación realizada por Lilo \& Martini (2013) tampoco se encuentran acorde a los obtenidos en el presente análisis, ya que su estudio determinó que en Latinoamérica lideran Brasil, Chile y Argentina.

En relación a las áreas de la Psicología acerca de las cuales se investiga, los resultados de la investigación llevada a cabo por García, Guerrero, Vargas \& Moya-Anegón (2008) arrojó que en los países latinoamericanos realizan generalmente investigaciones en Psicología orientadas al área de las Neurociencias, la Psicología Social y la Psicología Educativa. Sin embargo, dichas conclusiones no van en coherencia a la presente investigación, ya que al realizar el análisis correspondiente se encontró que las áreas de mayor interés para los autores son la Psicología Social, la Psicología Clínica y la Psicología Educativa.

El análisis realizado muestra una mayor tendencia de escogencia en desarrollar investigaciones a nivel de Psicología Clínica, como segundo foco de atención científica se encuentra el area a nivel social y como población principal de estudio se muestra variada y es seleccionada según el objetivo y planteamiento investigativo. Como autores pretendemos mostrar una ventana organizada y flexible de opciones a los lectores de la Revista Psicogente frente a las líneas de investigación tenida en cuenta durante estos 4 años de furor científico y en crecimiento.

\section{REFERENCIAS}

Abrahan, V. \& Justel, N. (2015). La improvisación musical. Una mirada compartida entre la musicoterapia y las neurociencias. Psicogente, 18(34), 372-384. https:// doi.org/10.17081/psico.18.31.512

Álvarez, L. (2014). Caracterización de las creencias irracionales del trastorno histriónico de la personalidad. Psicogente, 17(31), 143-154. https://doi. org/10.17081/psico.17.31.1475

Andrade, J., \& Gonzáles, J. (2017). Relación entre riesgo suicida, autoestima, desesperanza y estilos de socialización parental en estudiantes de bachillerato. Psicogente, 20(37), 70-88. https://doi.org/10.17081/psico.20.37.2419

Andreucci, P. \& Tartakowsky, V. (2016). La estrategia del sistema solar desde la perspectiva del sí mismo dialógico. Psicogente, 19(36), 311-323. https://doi. org/10.17081/psico.19.36.1300

Angeli, L. (2015). Reflexiones en torno a dos situaciones de conflicto vecinal y sus implicancias en patrones de interacción vinculados al género. Psicogente, 18(33), 129-140. https://doi.org/10.17081/psico.18.33.61

Arévalo, D. M. \& Cano, S. L. (2014). Estudio exploratorio sobre los intereses profesionales en estudiantes de la carrera de Psicología de la UCA. Psicogente, 17(31), 33-48. https://doi.org/10.17081/psico.17.31.1468 
Arias, A. \& Mazo, J. (2017). Sensibilidad ciudadana: sentidos y prácticas en jóvenes universitarios. Psicogente, 20(37), 135-145. http://doi.org/10.17081/ psico.20.37.2423

Arias, I., Cardoso, T., Aguirre, H. \& Arenas, J. (2016). Características psicológicas de rendimiento deportivo en deportes de conjunto. Psicogente, 19(35), 25-36. https://doi.org/10.17081/psico.19.35.1206

Arrial, Y. \& Neves, M. (2016). Escuela, homomaternidad y educación: reflexiones sobre las clases en la contemporaneidad. Psicogente, 19(35), 136-147. https:// doi.org/10.17081/psico.19.35.1214

Aurioles, I. \& Torres, T. (2016). Dimensiones culturales sobre seguridad y salud ocupacional de trabajadores de una empresa de manufactura prefarmacéutica. Psicogente, 19(36), 206-216. https://doi.org/10.17081/psico.19.36.1292

Austria, F. \& Castañeda, S. (2014). Construcción de una escala para medir relaciones de poder en el aula. Psicogente, 17(32), 337-351. http://revistas.unisimon.edu. co/index.php/psicogente/article/view/1448/1433

Barreira, I. (2015). El DSM, del nomenclador a la guía de tratamiento: Un comentario crítico a la nosografía americana. Psicogente, 18(33), 117-128. https://doi. org/10.17081/psico.18.33.60

Beltrán, C., Díaz, L., \& Zapata, M. (2017). Tamizaje del síndrome de Asperger en estudiantes de dos ciudades de Colombia a través de las escalas CAST y ASSQ. Psicogente, 20(38), 320-335. https://doi.org/10.17081/psico.20.38.2554

Betancourth, S., Burbano, D. \& Venet, M. (2017). La relación docente-estudiantes de preescolar según el CLASS de Pianta. Psicogente, 20 (37), 55-69. https://doi. org/10.17081/psico.20.37.2418

Buitrago, J., Pulido, L., \& Güicha-Duitama, Á. (2017). Relación entre sintomatología depresiva y cohesión familiar en adolescentes de una institución educativa de Boyacá. Psicogente, 20 (38) 296-307. https://doi.org/10.17081/psico.20.38.2550

Cabrera-Garcia, V., Casas, K., Pardo, S. \& Rodríguez, D. (2017). Análisis de la resiliencia en personas divorciadas, según su nivel educativo e ingresos económicos. Psicogente, 20(37), 160-171. https://doi.org/10.17081/psico.20.37.2425

Caldera, J., Aceves, B. \& Reynoso, O. (2016). Resiliencia en estudiantes universitarios. Un estudio comparado entre carreras. Psicogente, 19(36), 227-239. https://doi. org/10.17081/psico.19.36.1294

Calderón, J., Laca, F., Pando, M., \& Pedroza, F. (2015). Relación de la socialización organizacional y el compromiso organizacional en trabajadores mexicanos. Psicogente, 18(34), 267-277. https://doi.org/10.17081/psico.18.34.503

Calle, D. (2017). Filogenia y desarrollo de funciones ejecutivas. Psicogente, 20(38) 368-38. https://doi.org/10.17081/psico.20.38.2557

Campo, L. (2014). El desarrollo del autoconcepto en niños y niñas y su relación con la interacción social en la infancia. Psicogente, 17(31), 67-79. https://doi. org/10.17081/psico.17.31.1470

Campo-Arias, A., Herazo, E. \& Oviedo, H. (2017). Correlación entre actitud hacia homosexualidad femenina y masculina en estudiantes de Medicina. Psicogente, 20(37), 172-180. http://doi.org/10.17081/psico.20.37.2426

Cárdenas, T. \& Jaik, A. (2014). Inventario para la medición del Engagement (ilusión por el trabajo). Un estudio instrumental. Psicogente, 17(32), 294-306. https://doi. org/10.17081/psico.17.32.10

Cardona, M. \& Varela, V. (2017). Desempeño lector en niños con diagnóstico de TDAH (Trastorno de Déficit de Atención con Hiperactividad). Psicogente, 20(37), 99-117. https://doi.org/10.17081/psico.20.37.2421 
Cariaga, M. L \& Casari, L. (2014). La incidencia de rasgos de personalidad en estudiantes avanzados de Psicología hacia la elección de una línea teórica. Psicogente, 17(32), 379-396. https://doi.org/10.17081/psico.17.32.16

Castañeda, N. (2016). Prevención psicológica y neuropsicológica de factores de riesgo suicida en estudiantes universitarios. Psicogente, 19(36), 336-346. https:// doi.org/10.17081/psico.19.36.1302

Cerquera, A., Pabón, D., Granados, F. \& Galvis, M. (2016). Sobrecarga en cuidadores informales de pacientes con Alzheimer y la relación con su ingreso salarial. Psicogente, 19(36), 240-25. https://doi.org/10.17081/psico.19.36.1295

Cerquera, A., Peña, A., García, C., Orejuela, D. \& García, M. (2017). Psicología de la salud en el contexto del conflicto armado en Colombia: un marco de referencia. Psicogente, 20(37), 181-191. https://doi.org/10.17081/psico.20.37.2427

Cerquera, A., Uribe, A., Matajira, Y. \& Correa, H. (2017). Dependencia funcional y dolor crónico asociados a la calidad de vida del adulto mayor. Psicogente, 20(38), 398-409. https://doi.org/10.17081/psico.20.38.2561

Cervantes, M., Acosta, J., Balaguera, K., Valenzuela, A. \& Torres, A. (2014). Uso de alcohol en los estudiantes de tercero a quinto semestre del programa de Psicología de una universidad privada de la ciudad de Barranquilla. Psicogente, 17(32), 460-476. https://doi.org/10.17081/psico.17.32.23

Chahín-Pinzón, N. (2015). Consideraciones y reflexiones acerca de la versión colombiana de la escala Barratt de impulsividad para niños (BIS-11c). Psicogente, 18(34), 396-405. http://doi.org/10.17081/psico.18.34.514

Chávez, M., Petrzelová, J., Zapata, J., Hernández, M. \& Rodríguez, M. (2015). Violencia psicosocial en el noreste de México: repercusiones en la salud mental. Psicogente, 18(33), 11-21. https://doi.org/10.17081/psico.18.33.52

Collantes, B. I. \& Escobar, H. A. (2016). Desarrollo de la hipótesis como herramienta del pensamiento científico en contextos de aprendizaje en niños y niñas entre cuatro y ocho años de edad. Psicogente, 19(35), 77-97. https://doi.org/10.17081/ psico.19.35.1210

Córdoba-Paz, E. \& Betancourth-Zambrano, S. (2017). Consumo de sustancias psicoactivas en una universidad privada de Pasto, Colombia. Psicogente, 20(38), 308-319. https://doi.org/10.17081/psico.20.38.2552

Cruz, D. \& Puentes, A. (2017). Relación entre las diferentes dimensiones del síndrome de Burnout y las estrategias de afrontamiento empleadas por los guardas de seguridad de una empresa privada de la ciudad de Tunja. Psicogente, 20(38), 268-281. https://doi.org/10.17081/psico.20.38.2548

Diazgranados, P. \& Sánchez, D. (2014). Representación social del trabajo en jóvenes que culminan sus estudios de bachillerato en el distrito de Santa Marta. Psicogente, 17(32), 406-420.

Elvira-Valdés, M. \& Pujol, L. (2015). Propiedades psicométricas y estructura factorial de la escala de aprendizaje autorregulado (EAA) en adolescentes. Psicogente, 18(33), 66-77. https://doi.org/10.17081/psico.18.33.56

Enríquez, M., Fajardo, M. \& Garzón, F. (2015). Una revisión general a los hábitos y técnicas de estudio en el ámbito universitario. Psicogente, 18(33), 166-187. https://doi.org/10.17081/psico.18.33.64

Farias-Carracedo, C. \& Marlon, D. (2017). El "Estado Mamá": estudio histórico del Acogimiento Familiar en Mendoza (Argentina). Psicogente, 20(37), 36-54. http:// doi.org/10.17081/psico.20.37.2417

Fernández, J. \& Miralles, F. (2014). Formación en los trastornos de desarrollo. Utilización de metodología eLearning. Psicogente, 17(32), 283-293. 
Ferrel, F., Cuan, A., Londoño, Z., \& Ferrel, L. (2015). Factores de riesgo y protectores del bullying escolar en estudiantes con bajo rendimiento de cinco instituciones educativas de Santa Marta, Colombia. Psicogente, 18(33), 188-205. https://doi. org/10.17081/psico.18.33.65

Ferrel, F., Ferrel, L., Cantillo, A., Jaramillo, J. \& Jiménez, S. (2017). Variables académicas y sociodemográficas relacionadas con el Síndrome de Burnout, en estudiantes de Ingenierías y Ciencias de la Salud de una universidad estatal de Colombia. Psicogente, 20(38), 336-352. https://doi.org/10.17081/psico.20.38.2555

Fortes, I. \& Kother, M. (2017). Automutilação na adolescência - rasuras na experiência de alteridade. Psicogente, 20(38), 353-367. https://doi.org/10.17081/ psico.20.38.2556

Franco, M., Cárdenas, R. \& Santrich, E. (2016). Factores asociados a la comprensión lectora en estudiantes de noveno grado de Barranquilla. Psicogente, 19(36), 296-310. https://doi.org/10.17081/psico.19.36.1299

Gamboa, A., Ortiz, J. \& Muñoz, P. (2017). Violencia en contextos escolares: percepción de docentes sobre manifestaciones de violencia en instituciones educativas en Cúcuta-Norte de Santander. Psicogente, 20(37), 89-98. https://doi.org/10.17081/ psico.20.37.2420

Garassini, M., \& Valle, V. (2015). Aprecio a la belleza como fortaleza del carácter y creencias asociadas en adultos venezolanos. Psicogente, 18(33), 278-292. https:// doi.org/10.17081/psico.18.34.504

García, A., Guerrero, V., Vargas, B. \& Moya-Anegón, F. (2008). La psicología en el cienciograma de los países iberoamericanos. Revista Latinoamericana de Psicología, 40(3), 409-424. http://dx.doi.org/10.14349/rlp.v40i3.357

García, M. \& Forero, C. (2014). Motivación y satisfacción laboral como facilitadores del cambio organizacional: Una explicación desde las ecuaciones estructurales. Psicogente, 17(31), 120-142. https://doi.org/10.17081/psico.17.31.1474

García, P., Molerio, O. \& Pedraza, L. (2014). La prevención del tabaquismo y el alcoholismo en adolescentes y jóvenes desde las instituciones educativas. Psicogente, 17(31), 93-106. https://doi.org/10.17081/psico.17.31.1472

González, R., Hidalgo, G., León, S., Contreras, M., Aldrete, M., Hidalgo, B. y otros. (2015). Relación entre género y calidad de vida laboral en profesionales de salud. Psicogente, 18(33), 52-65. https://doi.org/10.17081/psico.18.33.55

Guerra, J., Guevara, C. y \& Robles, S. S. (2014). Validación del Inventario de Estrategias Metacognoscitivas y Motivación por la Lectura (IEMML) en estudiantes de Psicología. Psicogente, 17(31), 17-32. https://doi.org/10.17081/psico.17.31.1467

Guerrero-Martelo, M., Galván, G., Vásquez, F., Lázaro, G. \& Morales, D. (2015). Relación entre autoestima y autonomía funcional en función del apoyo social en adultos institucionalizados de la tercera edad. Psicogente, 18(33), 303-310. https://doi.org/10.17081/psico.18.34.506

Gutiérrez de Piñeres, C. (2017). Análisis de las prácticas de entrevistas forenses durante la etapa de investigación por denuncias de delitos sexuales en contra de niños, niñas y adolescentes. Psicogente, 20(37), 118-134. https://doi. org/10.17081/psico.20.37.2422

Guzmán, F. M. \& Vargas, D. E. (2014). Incidencia de la educación inicial en la construcción de subjetividad en niños y niñas de 3 a 5 años en situación de desplazamiento en un jardín en Bogotá en el sector de Bosa. Psicogente, 17(32), 307-322. https://doi.org/10.17081/psico.17.32.11

Henríquez, G., Rada, J. \& Jassir, E. (2016). Análisis del comportamiento del consumidor: medición cuantitativa del servicio en estudiantes de Administración de 
una universidad acreditada en Barranquilla. Psicogente, 19(36), 266-283. http:// doi.org/10.17081/psico.19.36.1297

Hernández, R., Fernández, C. \& Baptista, P. (2014). Metodología de la investigación. Quinta Edición. México. D.F: McGraw-Hill.

Iguarán, A., Anaya, C., Paba, C. \& Obispo, K. (2016). Confiabilidad y validez de la Escala de Observación de Estrategias Cognitivas y Metacognitivas (EOECM) para la evaluación de la metacognición y la atención en niños de preescolar. Psicogente, 19(35), 98-109. https://doi.org/10.17081/psico.19.35.1211

Juárez, D. M. \& Landero, R. (2014). Ritmos circadianos en pacientes oncológicos. Psicogente, 17(32), 352-364.

Labín, A., Taborda, A. \& Brenlla, M. (2015). La relación entre el nivel educativo de la madre y el rendimiento cognitivo infanto-juvenil a partir del WISC-IV. Psicogente, 18(34), 293-302. http://doi.org/10.17081/psico.18.34.505

Lillo, S. \& Martini, N. (2013). Principales Tendencias Iberoamericanas en Psicología Clínica. Un Estudio Basado en la Evidencia Científica. Terapia Psicológica, 31(3), 363-371. http://dx.doi.org/10.11144/Javeriana.upsy14-3.pcah

López-López, W., Silva, L., García-Cepero, M., Aguilar-Bustamante, M., \& AguadoLópez, E. (2011). Retos para la colaboración nacional e internacional en la psicología latinoamericana: un análisis del sistema RedALyC, 2005-2007. Estudios de Psicología, 16(1), 17-22. http://hdl.handle.net/20.500.11799/38722

Luna, A. C. A. \& Laca, F. V. (2014). Análisis factorial confirmatorio de la Escala de Satisfacción con la Vida de Familia (ESVF) en adolescentes de secundaria y bachillerato. Psicogente, 17(31), 226-240. https://doi.org/10.17081/psico.17.31.1480

Madariaga, C. \& Lozano, J. E. (2016). El apoyo social en estudiantes universitarios y su relación con las comunicaciones cara a cara y las comunicaciones mediadas por las Tecnologías de la Información y la Comunicación (TIC). Psicogente, 19(35), 47-62. http://doi.org/10.17081/psico.19.35.1208

Marín, J. (2014). Conductas prosociales en los barrios Modelo y Los Trupillos de Barranquilla. Psicogente, 17(31), 211-225. https://doi.org/10.17081/psico.17.31.1479

Martínez, R., Pando, M., León, S., González, R., Aldrete, M. \& López, L. (2015). Validez y confiabilidad de la escala de salud mental positiva. Psicogente, 18(33), 78-88. http://doi.org/10.17081/psico.18.33.57

Mass, L. (2014). El sujeto y la estética corporal en la sociedad contemporánea (algunas relaciones teóricas con el capitalismo y plus de gozar). Psicogente, 17(31), 155-162. http://revistas.unisimon.edu.co/index.php/psicogente/article/ view/1476/1461

Mebarak, M., De Castro, A., Polo, J., Morales, M., Villabona, D., Rodriguez, J. y otros. (2017). Estudio descriptivo de las autopsias en los departamentos Atlántico y Magdalena. Psicogente, 20(37), 12-24. https://doi.org/10.17081/ psico.20.37.2415

Montiel, V. \& Guerra, V. (2016). Aproximaciones teóricas sobre Psicoeducación. Análisis de su aplicación en mujeres con cáncer de mama. Psicogente, 19(36), 324-335. https://doi.org/10.17081/psico.19.36.1301

Morales, G. (2015). Diversidad auditiva: imaginarios sociales e inclusión laboral: Una aproximación intercultural. Psicogente, 18(34), 364-371. http://revistas. unisimon.edu.co/index.php/psicogente/article/view/1428/2630

Morales, Y. (2014). Inclusión social de los vendedores que ejercen actividades laborales del sector informa en el Centro Histórico de Barranquilla. Psicogente, 17(31), 190-210. http://revistas.unisimon.edu.co/index.php/psicogente/article/ view/1478/1463 
Moral, J. \& Segovia, M. (2015). Discriminación en la familia a mujeres que viven con VIH. Psicogente, 18(33), 89-103. https://doi.org/10.17081/psico.18.33.58

Moreno, G. \& Mantilla, J. (2016). Una revisión del concepto observatorio social: hacia una comprensión de sus objetivos, alcances, métodos y finalidades. Psicogente, 19(36), 347-359. https://doi.org/10.17081/psico.19.36.1303

Moreno, G., Rojas, L., Fagua, A., Sanabria, H., Sanabria, M. \& Rey, N. (2015). Subjetividades y territorialidades: una aproximación desde la cartografía social en el contexto colombiano. Psicogente, 18(33), 206-225. http://doi.org/10.17081/ psico.18.33.66

Moreno, J. H., Hernández, D. \& Suárez, M. A. (2016). Análisis bibliométrico de los problemas de comportamiento externalizado en niños y adolescentes de la ciudad de Bogotá (2003-2013). Psicogente, 19(35), 63-76. https://doi.org/10.17081/ psico.19.35.1209

Moretti, M. \& Casari, L. (2017). Satisfacción y motivación de la cirugía estética en mujeres y su relación con los esquemas desadaptativos tempranos. Psicogente, 20(38), 222-239. https://doi.org/10.17081/psico.20.38.2543

Muñoz, M. (2014). Diseño y evaluación de un programa de prevención de consumo de alcohol en una institución universitaria diseñado con los estudiantes. Psicogente, 17(32), 365-378. http://revistas.unisimon.edu.co/index.php/psicogente/ article/view/1450

Obando, L., Salcedo, M. \& Correa, L. (2017). La atención psicosocial a personas víctimas del conflicto armado en contextos institucionales de salud pública. Psicogente, 20(38), 382-397. https://doi.org/10.17081/psico.20.38.2559

Orozco, A. (2014). Impacto social y académico del Programa Interacción Social y Desarrollo Ciudadano en tres localidades del departamento del Atlántico. Psicogente, 17(32), 477-494. http://revistas.unisimon.edu.co/index.php/psicogente/ article/view/1459

Ortega, M. (2016). Los directores aprendiendo de sus maestros. Psicogente, 19(36), 217-226. https://doi.org/10.17081/psico.19.36.1293

Páramo, P., Hederich, C., López, O., Sanabria, L. \& Camargo, A. (2015). ¿Dónde ocurre el aprendizaje? Psicogente, 18(34), 320-335. https://doi.org/10.17081/ psico.18.34.508

Pardo, L., Bernal, C., Ávila, D. \& Muñoz, L. (2016). Análisis psicométrico del Cuestionario de Motivos del Consumo de Alcohol (CMC) en escolares de la ciudad de Bogotá. Psicogente, 19(36), 284-295. http://doi.org/10.17081/psico.19.36.1298

Paredes, Y. (2015). Factores psicosociales y desnutrición crónica en niños y niñas escolarizados. Psicogente, 18(33), 385-395. https://doi.org/10.17081/ psico.18.34.513

Parra, A. \& Corbetta, J.M. (2014). Examen de post-efecto para experiencias anómalo/ paranormales con un abordaje humanista. Psicogente, 17(32), 397-405. http:// revistas.unisimon.edu.co/index.php/psicogente/article/view/1452/1437

Pérez, M. (2015). Alcances de las políticas nacionales de ciencia, tecnología e innovación en el departamento del Atlántico. Psicogente, 18(34), 406-419. http:// doi.org/10.17081/psico.18.34.515

Pérez, M.P., Vianchá, M.A., Martínez, L.C. \& Salas, I.C. (2014). El maltrato familiar y su relación con la ideación suicida en adolescentes escolarizados de instituciones públicas y privadas de las ciudades de Tunja, Duitama y Sogamoso. Psicogente, 17(31), 80-92. https://doi.org/10.17081/psico.17.31.1471 
Pérez, Y.E. \& Otero, I. (2014). Calidad de vida, ansiedad, depresión y optimismo disposicional en pacientes con lupus eritematoso. Psicogente, 17(31), 107-119. http://revistas.unisimon.edu.co/index.php/psicogente/article/view/1473

Pinilla, V. E., Montoya, D., \& Dussán, C. (2015). Caracterización del Autoconcepto en una muestra de estudiantes universitarias del Programa de Desarrollo Familiar de Manizales, Colombia. Psicogente, 18(33), 141-156. https://doi.org/10.17081/ psico.18.33.62

Pino, M. (2015). Rendimiento cognitivo de adolescentes con VIH en pruebas para la exploración de la flexibilidad mental. Psicogente, 18(33), 157-165. http://doi. org/10.17081/psico.18.33.63

Polo, J. D., Santiago, V., Navarro, M. C. \& Alí, A. (2016). Creencias irracionales, síndrome de Burnout y adicción al trabajo en las organizaciones. Psicogente, 19(35), 148-16. https://doi.org/10.17081/psico.19.35.1215

Ramírez, A.F. \& Arroyo, K. (2014). Características neuropsicológicas en adolescentes infractores de la ciudad de Sincelejo-Sucre. Psicogente, 17(32), 421-430. http:// revistas.unisimon.edu.co/index.php/psicogente/article/view/1454/1439

Redondo, J., Rangel, K., \& Luzardo, M. (2015). Diferencias en comportamientos prosociales entre adolescentes colombianos. Psicogente, 18(33), 311-319. https://doi. org/10.17081/psico.18.34.507

Rey-Anacona, C., Martínez-Gómez, J., \& Guerrero-Rodríguez, S. (2009). Tendencias de los Artículos en Psicología Clínica en Iberoamérica. Terapia Psicológica, 27(1), 61-71. http://biblioteca.universia.net/html_bura/ficha/params/title/tendencias-articulos-psicologia-clinica-iberoamerica/id/54646726.html

Reyes, L., Alarcón, Y., \& Bahamón, M. (2014). Tendencias en las líneas de investigación de Doctorados en Psicología de universidades iberoamericanas. Psicogente, 17(32), 442-451. http://revistas.unisimon.edu.co/index.php/psicogente/ article/view/1456

Reyes, V., Reséndiz, A., Alcázar, R., \& Reidl, L. (2017). Las estrategias de afrontamiento que utilizan los adolescentes ante situaciones que provocan miedo. Psicogente, 20(38), 240-255. https://doi.org/10.17081/psico.20.38.2544

Rico, F. \& Puentes, P. (2016). Las neurociencias para el abordaje de la didáctica de las finanzas. Psicogente, 19(35), 161-176. https://doi.org/10.17081/psico.19.35.1216

Rincón, G., Hernández, C., \& Prada, R. (2017). Influencia de los movimientos sacádicos en el rendimiento académico de estudiantes de básica primaria en situación de vulnerabilidad en la ciudad de Cúcuta. Psicogente, 20(38), 256-267. https://doi. org/10.17081/psico.20.38.2545

Rivera, A. (2016). Culturas juveniles y estrategias de aprendizaje en educación superior, perspectiva intercultural. Psicogente, 19(35), 128-135. http://doi. org/10.17081/psico.19.35.1213

Robles, E., Oudhof, H. \& Mercado, A. (2016). Validez y confiabilidad del instrumento de vínculo parental (Parental Bonding Instrument, PBI) en una muestra de varones mexicanos. Psicogente, 19(35), 14-24. https://doi.org/10.17081/ psico.19.35.1205

Rodríguez, M.T., Roque, Y. \& Rodríguez, M. (2014). Desarrollo de la grupalidad, mediado por las TIC, en jóvenes universitarios. Psicogente, 17(31), 163-173. http://revistas.unisimon.edu.co/index.php/psicogente/article/view/1477

Rodríguez, U., Trillos, L.F. \& Baute, V.C. (2014). Caracterización del síndrome de Burnout en amas de casa residentes en el barrio Rodrigo de Bastidas de la ciudad de Santa Marta, Colombia. Psicogente, 17(32), 431-441. http://www.scielo.org. co/pdf/psico/v17n32/v17n32a14.pdf 
Romero, K., Rojas, M., Abad, A. \& Gómez, G. (2016). ¿Afecta el virus del chikungunya el estado emocional de los individuos que lo padecen? Psicogente, 19(35), 37-46. http://doi.org/10.17081/psico.19.35.1207.

Salcedo, M. \& Ortiz, A. (2014). Aplicabilidad de la teoría de sistemas autorreferentes de Niklas Luhmann al pensamiento humano. Psicogente, 17(32), 269-282. https:// doi.org/10.17081/psico.17.32.8

Salcedo, M. (2015). La cuestión de lo político en el psicoanálisis. Psicogente, 18(34), 420-432. https://doi.org/10.17081/psico.18.34.516

Sanz, A., Moreno, M. \& Pérez, R. (2016). Significaciones del proceso de integración social de jóvenes exinfractores en la ciudad de Cali. Psicogente, 19(35), 110-127. https://doi.org/10.17081/psico.19.35.1212

Serrano, J. \& Angarita, B. (2016). Estudio comparativo del estado cognitivo para el aprendizaje en niños y niñas de instituciones educativas públicas del departamento del Cauca, Colombia. Psicogente, 19(36), 252-265. https://doi. org/10.17081/psico.19.36.1296

Sosa, F., \& Zubieta, E. (2015). La experiencia de migración y adaptación sociocultural: identidad, contacto y apoyo social en estudiantes universitarios migrantes. Psicogente, 18(33), 36-51. http://dx.doi.org/10.17081/psico.18.33.54

Spiendler, S., Carlotto, M., Ogliari, D., \& Giordani, K. (2015). Etressores ocupacionais em psicólogos clínicos brasileiros. Psicogente, 18(33), 104-116. https://doi. org/10.17081/psico.18.33.59

Toro, R. A. \& Vargas, C.V. (2014). Escala de Sociotropía-Autonomía (SAS): propiedades psicométricas de la adaptación a Colombia. Psicogente, 17(32), 323-336. https:// doi.org/10.17081/psico.17.32.12

Torres, P. \& Granados, D. E. (2014). Procesos cognoscitivos implicados en la comprensión lectora en tercer grado de educación primaria. Psicogente, 17(32), 452-459. http://revistas.unisimon.edu.co/index.php/psicogente/article/ view/1457

Trujillo, S. (2015). La moral corrige a los instintos y el amor corrige a la moral. Psicogente, 18(34), 433-437. https://doi.org/10.17081/psico.18.34.517

Uribe, J., Riaño, M., Bonilla, N., Carrillo, S., Hernández, Y., \& Bahamón, M. (2017). Percepción de autoeficacia vs. rechazo del uso del condón en las prácticas sexuales de mujeres y hombres jóvenes. Psicogente, 20(37), 25-35. https://doi. org/10.17081/psico.20.37.2416

Valdez, M. \& Iranzo, M. (2014). Ejes teórico-conceptuales del capital social en ámbitos rurales. Psicogente, 17(31), 49-66. https://doi.org/10.17081/psico.17.31.1469

Vásquez, É. (2017). Estudio de las conductas prosociales en niños de San Juan de Pasto. Psicogente, 20(38), 282-295. https://doi.org/10.17081/psico.20.38.2549

Vera-Villarroel, P., López-López, W., Lillo, S. \& Silva, L. (2011). La producción científica en psicología latinoamericana: Un análisis de la investigación por países. Revista Latinoamericana de Psicología, 43(1), 95-104. http://dx.doi.org/10.14349/rlp. v43i1.613

Vizcaíno, A. \& Manzano, M. (2015). Relación entre creencias epistemológicas sobre la matemática y métodos de enseñanza en profesores de esta asignatura en la secundaria básica. Psicogente, 18(33), 22-35. http://dx.doi.org/10.17081/ psico.18.33.53

Yáñez, H. \& Ortíz, A. (2015). Exploración del uso de las competencias académicas definidas en el proceso educativo y formativo en Psicología. Psicogente, 18(34), 336-350. https://doi.org/10.17081/psico.18.34.509 
Zambrano, C., Aroca, L. \& Rosero, D. (2015). Autogestión en la red de agentes comunitarios en salud mental en las instituciones educativas del municipio de San Lorenzo (Nariño). Psicogente, 18(34), 351-363. http://doi.org/10.17081/ psico.18.34

Zambrano, C., Perugache, A. \& Figueroa, J. (2017). Manifestaciones de la violencia basada en género en docentes universitarios. Psicogente, 20(37), 146-159. http:// doi.org/10.17081/psico.20.37.242 